OPEN ACCESS

Edited by: Daiij Kawanami, Fukuoka University, Japan

Reviewed by: Hisashi Yokomizo, Kyushu University, Japan Sima Lakdizaii, Tabriz Nursing \& Midwifery Faculty,

Iran

${ }^{*}$ Correspondence: Moritsugu Kimura moritsug@is.icc.u-tokai.ac.jp

Specialty section: This article was submitted to Diabetes Self-Management, a section of the journal

Frontiers in Clinical

Diabetes and Healthcare

Received: 02 December 2021 Accepted: 14 December 2021 Published: 26 January 2022

Citation:

Kimura M, Toyoda M, Saito N, Abe M, Kato E, Sugihara A, Ishida N and Fukagawa M (2022) A Survey on the Current Status of Ophthalmological

Consultations in Patients With Diabetes Undergoing Maintenance Hemodialysis and the Effectiveness of Education on Consultation

Behavior-Experience of a Single Hemodialysis Clinic in Japan. Front. Clin. Diabetes Healthc. 2:827718. doi: 10.3389/fcdhc.2021.827718

\section{A Survey on the Current Status of Ophthalmological Consultations in Patients With Diabetes Undergoing Maintenance Hemodialysis and the Effectiveness of Education on Consultation Behavior -Experience of a Single Hemodialysis Clinic in Japan}

\author{
Moritsugu Kimura ${ }^{1,2 *}$, Masao Toyoda ${ }^{1,2}$, Nobumichi Saito ${ }^{2}$, Makiko Abe ${ }^{2}$, Eri Kato ${ }^{1}$, \\ Akemi Sugihara $^{1}$, Naoto Ishida ${ }^{1}$ and Masafumi Fukagawa ${ }^{2}$ \\ 1 Seichi Clinic, Isehara, Japan, ${ }^{2}$ Division of Nephrology and Metabolism, Department of Internal Medicine, Tokai University \\ School of Medicine, Isehara, Japan
}

Introduction: It is extremely important for patients with diabetes undergoing maintenance hemodialysis (MHD) to receive regular ophthalmologic examinations. However, even in the field of MHD in Japan, where there are many hemodialysis patients and the survival rate is said to be one of the highest in the world, we often see patients with diabetes who do not receive regular ophthalmologic examinations. In this study, we surveyed the status of ophthalmology consultations and the use of diabetic eye notebook (DEN) among hemodialysis patients with diabetes at hemodialysis clinics to confirm the current situation, with the aim of confirming the effectiveness of education on consultation behavior by medical care staff.

Materials and Methods: This study included 38 diabetic hemodialysis patients attending one MHD clinic in Japan for one year from March 2018 to March 2019. In the first fact-finding survey in March 2018, hemodialysis care unit nurses (HCUNs) in the hemodialysis unit asked the diabetic hemodialysis patients whether they had consulted an ophthalmologist and used the DEN. Based on the results, the HCUNs recommended that hemodialysis patients with complications of diabetes be educated about the usefulness of regular ophthalmologic examinations, even during MHD, and that they use the DEN. This was followed by a second fact-finding survey in March 2019 to reconfirm ophthalmology consultations and DEN use.

Results: Regarding the presence of ophthalmology consultations, 22 of 38 (58\%) patients had regular ophthalmology consultations in March 2018, and 27 of 38 (71\%) patients had consultations in the following year after receiving information from an HCUN. 
Only 1 of 22 patients (5\%) who consulted the ophthalmologist in March 2018 used a DEN, but 19 of 27 patients (70\%) used it the following year.

\begin{abstract}
Conclusion: In the future, the development and utilization of a new DEN that includes more detailed patient information, and the spread of self-care guidance to patients by multidisciplinary health care professionals, will increase the consultation rate of MHD patients in Japan and reduce the incidence and progression of ocular diseases in MHD patients.
\end{abstract}

Keywords: ophthalmological consultation, diabetic patient, hemodialysis, education, patient involvement, self-care

\section{INTRODUCTION}

End-stage renal disease (ESRD) has become an emerging health problem worldwide. The eye shares striking developmental, structural and genetic pathways with the kidney, suggesting that kidney disease and ocular disease may be closely related (1).

In particular, retinopathy affects the progression of nephropathy in patients with diabetic nephropathy, and it is said that early detection and management of patients with retinopathy is important to reduce the risk of death in patients with diabetic nephropathy (2-4). Patients with ESRD are at risk for developing ocular disease. This risk is associated with comorbidities that are common in ESRD patients, as well as to the unique effects of hemodialysis and the uremic state, which can lead to changes in the conjunctivae, cornea, retina, and macula. The most common ophthalmological complaints in ESRD patients include redness, irritation of the eyes, which may be associated with elevation of the product of the serum calcium and phosphorus concentrations, the so-called calciumphosphorus product or $\mathrm{Ca} \times \mathrm{P}$. In patients with chronically elevated calcium-phosphate products, band keratopathy may result. Other ophthalmological symptoms include retinal hemorrhage, ischemic optic neuropathy, ophthalmological infection, elevated intraocular pressure, retinal detachment, and macular edema. Prompt recognition that these conditions may threaten a patient's vision is required $(5,6)$.

Moreover, ESRD patients with diabetes mellitus undergoing hemodialysis have a higher incidence of ocular diseases, including diabetic retinopathy (DR), exudative retinal detachment, which can cause a major cause of decreasing visual acuity and blindness (711). Therefore, it is very important for patients with diabetes undergoing MHD to have regular ophthalmologic examinations. However, even in the field of MHD in Japan, which has a large number of hemodialysis patients and where the survival rate is said to be among of the highest in the world (12), we often see patients with diabetes who do not receive regular ophthalmologic examinations $(12,13)$. In addition, collaboration between physicians and ophthalmologists is said to be important in the treatment of diabetes in Japan. Figures 1A, B shows a diabetic eye notebook (DEN), the DEN was published by the Japanese Society of Ophthalmic Diabetology in 2002 as one of the solutions to this problem, patients are recommended to use this diary to consult ophthalmologist regularly (14). This study aimed to reduce the incidence and progression of ocular diseases in MHD patients by improving the ophthalmology consultation rate of MHD patients with diabetes, and we surveyed the status of ophthalmology consultations and the use of the DEN among patients with diabetes undergoing MHD at single hemodialysis clinic in order to confirm the current situation and discuss the need for medical collaboration between hemodialysis clinics and ophthalmology clinics and the educational activities for medical staff.

\section{MATERIALS AND METHODS}

The subjects were 38 patients (males: $n=25$, females: $n=13$ ) among 46 diabetic MHD patients who attended the Sechi Clinic (Isehara City, Kanagawa Prefecture) in March 2018, who continued to attend the clinic until March 2019, excluding patients who were transferred or died. In the first fact-finding survey in March 2018, hemodialysis care unit nurses (HCUNs) asked patients with diabetes undergoing MHD whether they were consulting the ophthalmological clinic and using a DEN. Based on the results, HCUNs verbally explained the three major complications of diabetes to MHD patients with diabetes, educated them on the usefulness of regular ophthalmologic consultations even during MHD because of the risk of blindness, and recommended the use of DEN. The third edition of the DEN was used, and the ophthalmologist's name, date of consultation, date of next consultation, corrected vision, etc. were recorded by the ophthalmologist. In addition, if there were any diseases, the status, changes, and treatment details were also recorded in "consultation notes" (Figure 1B). In the second fact-finding survey in March 2019, as in the first survey, the HCUNs asked MHD patients if they were consulting ophthalmologists and if they were using their DEN. From the medical records of 38 subjects, we also confirmed the history of ophthalmological consultation and the diagnosis of ocular diseases when they were referred to Seichi Clinic for MHD. This is a descriptive study of MHD patients who were followed up for one year after HCUNs awareness campaign.

\section{RESULTS}

The mean age of the 38 patients was 68.7 years and the mean duration of hemodialysis was 7.2 years. Diabetes mellitus was 


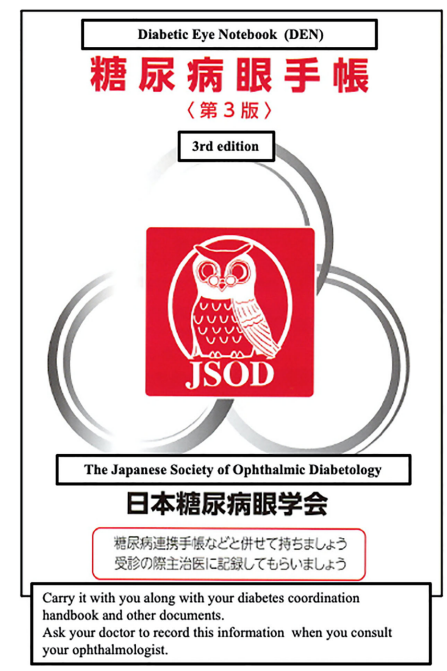

B

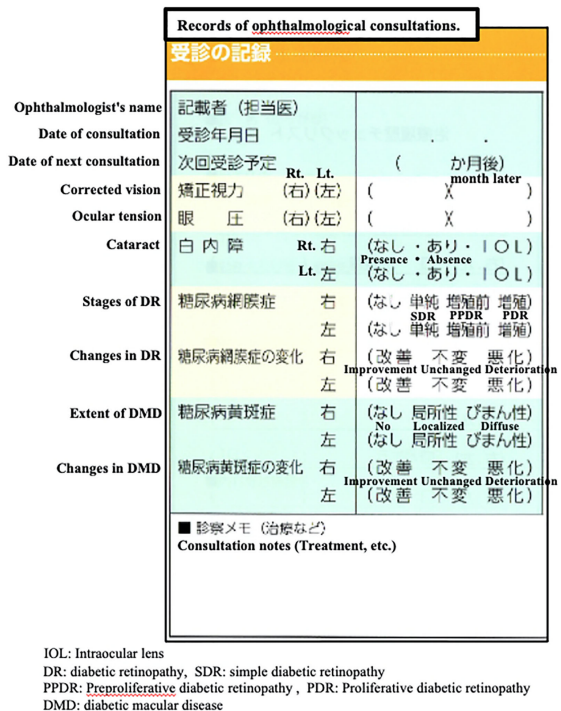

FIGURE 1 | (A) The cover of the diabetic eye notebook (DEN). (B) The detailed contents of the diabetic eye notebook (DEN).

treated with diet alone in 6 cases. The others received insulin therapy or GLP-1 receptor agonist therapy, or some oral hypoglycemic agents (Table 1). Regarding the diagnoses of ocular disease listed on the referral letters of the 38 patients who started MHD at the Seichi Clinic, 1 patient had no ocular disease, in 5 patients, the ocular disease status was unknown, and 32 patients had some kind of ocular disease. Of the 32 patients with confirmed ocular disease, 11 did not consult an ophthalmologist. The most common ocular diseases were DR in 19 patients, cataract in 17 patients, others in 5 patients (Table 2). Regarding the presence of ophthalmology consultations, 22 of 38 (58\%) patients had regular ophthalmology consultations in March 2018, and 27 out of 38 (71\%) patients had consultations in the following year after the awareness campaign (Figure 2). Only 1 of 22 patients (5\%) who consulted the ophthalmologist in March 2018 used the DEN, but 19 of 27 patients (70\%) used it the following year (Figure 3).

TABLE 1 | Clinical parameters of 38 patients.

\section{Patient Background}

Age (years)

$\operatorname{Sex}(M / F)$

Duration of dialysis (years)

$\mathrm{Hb}(\mathrm{g} / \mathrm{dL})$

$\mathrm{Ht}(\%)$

$\mathrm{HbA} 1 \mathrm{c}(\%)$

$\mathrm{GA}(\%)$

Insulin/GLP-1 receptor agonist (patients)

Oral medication DPP-4 inhibitor/glinide/ $\alpha-G I$ (patients)

Diet therapy only (patients)

\section{DISCUSSION}

In this study, HCUNs explained the necessity of ophthalmologic examinations to patients at a MHD clinic, which resulted in a $10 \%$ increase in the ophthalmological examination rate from approximately $60 \%$ to approximately $70 \%$ after one year, and the utilization rate of DEN increased significantly from $5 \%$ to $70 \%$. The reason why the HCUNs were able to achieve these results after only one year of educational activities may be that the effects of the collaboration between the physician, HCUNs and ophthalmologist before the introduction of MHD remained. However, considering the fact that $30 \%$ of patients had still not consulted ophthalmological clinics and that $30 \%$ of patients who did consult a clinic did not use the DEN. This may be due to the following problems: 1) dialysis physicians do not cooperate with ophthalmologists or physicians before the introduction of hemodialysis, and 2) self-care education for hemodialysis patients is not as complete as it was before the introduction of dialysis.

\section{1) Dialysis Physicians Do Not Cooperate With Ophthalmologists or Physicians Before the Introduction of Hemodialysis}

The fact that 11 of the 32 patients who had consulted an ophthalmologist before starting hemodialysis and who had been diagnosed with some type of ocular disease did not consult an ophthalmologist (Table 2) suggests that the physician and ophthalmologist may have collaborated prior to the start of dialysis, but that the dialysis physician may have stopped working with the ophthalmologist once dialysis started. In Japan, the importance of collaboration between physicians and ophthalmologists from the early stages of diabetes has been reported for some time $(15,16)$, 
TABLE 2 | Presence or absence of ophthalmological examination, use of DEN and the diagnoses of ocular disease listed on the referral letters of the 38 patients who started MHD.

\begin{tabular}{|c|c|c|c|c|c|c|c|}
\hline No & Ocular disease & Ophthalmological examination & Using DEN & No & Ocular disease & Ophthalmological examination & Using DEN \\
\hline 1 & PDR & 0 & $\times$ & 20 & $\mathrm{DR}$ & 0 & $\times$ \\
\hline 2 & Cataract & $\times$ & $\times$ & 21 & $\mathrm{DR}$ - Cataract & 0 & $\times$ \\
\hline 3 & None & 0 & $\times$ & 22 & Unknown & $\times$ & $\times$ \\
\hline 4 & PPDR & $x$ & $\times$ & 23 & PDR $\mathbf{~ C a t a r a c t ~} \mathbf{~}$ Other & 0 & $\times$ \\
\hline 5 & SDR & O & $\times$ & 24 & $\mathrm{DR}$ & 0 & $\times$ \\
\hline 6 & PPDR & 0 & $x$ & 25 & Unknown & $\times$ & $\times$ \\
\hline 7 & $\mathrm{DR}$ & 0 & $\times$ & 26 & Cataract & 0 & $\times$ \\
\hline 8 & PDR - Cataract - Other & 0 & 0 & 27 & Cataract & $x$ & $\times$ \\
\hline 9 & PDR • Other & 0 & $\times$ & 28 & Cataract & 0 & $\times$ \\
\hline 10 & DR $=$ Cataract & $x$ & $\times$ & 29 & Cataract & $x$ & $\times$ \\
\hline 11 & $\mathrm{DR}$ - Cataract & $\bigcirc$ & $\times$ & 30 & $\mathrm{DR}$ & $\times$ & $\times$ \\
\hline 12 & Unknown & $x$ & $\times$ & 31 & Other & $\times$ & $\times$ \\
\hline 13 & Cataract & 0 & $\times$ & 32 & Cataract & $\times$ & $x$ \\
\hline 14 & Cataract & 0 & $x$ & 33 & Cataract & 0 & $x$ \\
\hline 15 & PDR & 0 & $\times$ & 34 & Cataract & $x$ & $\times$ \\
\hline 16 & DR & 0 & $x$ & 35 & $\mathrm{DR}$ & 0 & $\times$ \\
\hline 17 & Other & $\times$ & $x$ & 36 & Unknown & $x$ & $\times$ \\
\hline 18 & Unknown & $\times$ & $x$ & 37 & DR & $\times$ & $x$ \\
\hline 19 & Cataract & 0 & $\times$ & 38 & DR - Cataract & 0 & $x$ \\
\hline
\end{tabular}

$D R$, diabetic retinopathy; SDR, simple diabetic retinopathy, PPDR, preproliferative diabetic retinopathy; PDR, proliferative diabetic retinopathy; DEN, diabetic eye notebook.

$\circ$ : The patient had an ophthalmologicalexamination. The patient used DEN.

x: The patient did not have an ophthalmological examination. The patient did not use DEN.

and in 2002, the Japanese Diabetic Eye Society issued the DEN, which recommends regular consults to ophthalmologists, as a means of collaboration (14). On the other hand, there are no reports on collaboration between dialysis doctors and ophthalmologists. However, considering the fact that dialysis patients are at high risk for the development of various ocular diseases $(5,6)$, it is important for dialysis physicians along with HCUNs to collaborate more closely with ophthalmologists using collaboration tools such as the DEN, and more case reports are expected. In addition, while the DEN is an effective handbook for collaboration with ophthalmologists, it does not have a column for important patient information such as weight, blood glucose, lipid profile, or liver/renal function, etc., even though there is a column for HbAlc levels. This information can only be provided in the "Consultation notes" section (Figure 1B). In addition, in the current 4th edition, the "Consultation notes" section has been deleted, making it difficult to describe additional information. In order for ophthalmologists, physicians, dialysis physicians and HCUNs to better collaborate on patient $1^{\text {st }}$ survey $(n=38)$

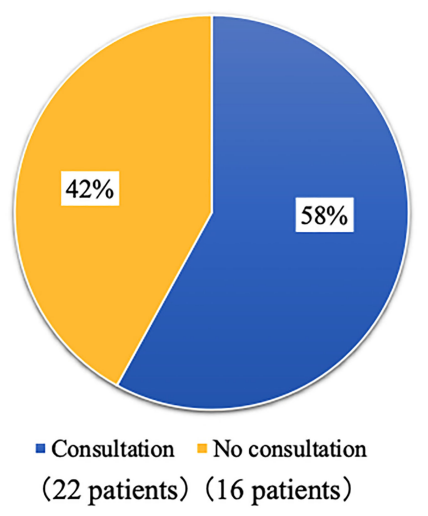

$2^{\text {nd }}$ survey $(\mathbf{n}=38)$

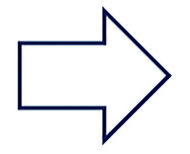

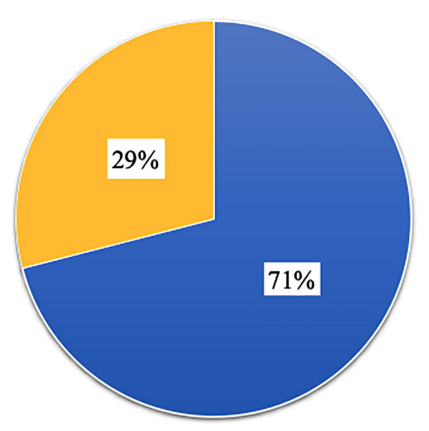

- Consultation $=$ No consultation (27 patients) (11 patients)

FIGURE 2 | The changes in the presence of ophthalmology consultations before and after the awareness campaign. Regarding the presence of ophthalmology consultations, 22 of 38 (58\%) patients had regular ophthalmology consultations in March 2018 (1st survey: the first fact-finding survey in March 2018 ), and 27 of 38 (71\%) patients had consultations in the following year after the awareness campaign (2nd survey: the second fact-finding survey in March 2019). 


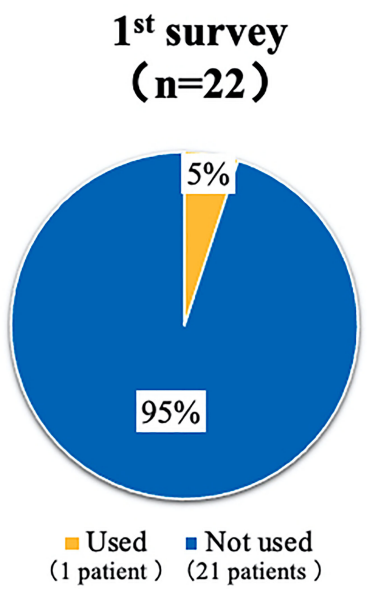

(1 patient) (21 patients )

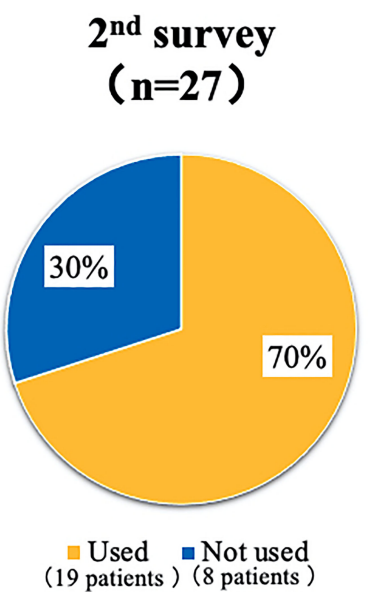

FIGURE 3 | The changes in the presence of the diabetic eye notebook (DEN) use before and after the awareness campaign. Only 1 of 22 patients (5\%) who consulted the ophthalmologist in March 2018 (1st survey: the first fact-finding survey in March 2018), used the diabetic eye notebook (DEN), but 19 of 27 patients (70\%) used it the following year (2nd survey: the second fact-finding survey in March 2019).

information in the future, it may be necessary to develop a new DEN that can describe this information.

In other countries, it is said that a partnership between primary care physicians and ophthalmologists is the only way to save many people at risk of diabetic retinopathy. Furthermore, in Japan, when diabetic nephropathy transitions to end-stage renal failure, the doctor in charge is often changed from a diabetologist to a nephrologist or a dialysis specialist (15). Under these circumstances, the use of a handbook such as DEN as a tool for understanding the clinical course of eye disease and for continuing cooperation with ophthalmologists is expected to help maintain the quality of life of many diabetic dialysis patients in Japan and abroad.

\section{2) Self-Care Education for Hemodialysis Patients Is Not as Complete as It Was Before the Introduction of Hemodialysis. Importance of Education and Team Care for Patients With Diabetes Undergoing MHD} Japan has very few kidney transplants and many MHD patients in comparison to the prevalence of ESRD (12). In addition, the survival rate of MHD patients is one of the highest in the world (13). Japanese clinical practice patterns differ from those of other countries in many ways, including the reasons for the longevity of MHD patients, as reported in the DOPPS (Dialysis Outcomes and Practice Patterns Study) study $(17,18)$.

This is reflected by the fact that the rate of ophthalmologic examinations and the use of DEN significantly increased only one year after the initiation of the awareness campaign by the HCUNs; however, the results of the first survey confirmed the fact that there has been a lack of awareness-raising activities and patient self-care education for ophthalmologic examinations. In recent years, "patient involvement in healthcare" has been attracting international attention as a way to achieve safe, high-quality healthcare. Patient involvement in healthcare means that patients and their families collaborate with medical professionals to improve the quality and safety of medical care, and the modes of participation are said to include a wide range of areas, not only at the level of the individual patient (e.g., decisions about treatment choices and self-care) but also in relation to hospital management and other areas (19-22). In this focus on the importance of patient involvement in healthcare, the importance of self-care education for diabetes has long been reported in Japan $(23,24)$, and steps have been taken to allow reimbursement for patient education and guidance to prevent dialysis. However, the importance of self-care education after the initiation of hemodialysis has not been emphasized as much. Through this survey, it was confirmed patients with diabetes require thorough self-care education before and after the initiation of hemodialysis in order to enhance patient involvement in healthcare. The hemodialysis unit team in Japan is usually composed of several healthcare professionals (e.g., nurses, clinical engineers, dieticians, and doctors) (25). If all of these medical professionals involved in dialysis understand the existence of DEN and can provide the same self-care guidance to patients as they did before the introduction of dialysis, it is estimated that more MHD patients will consult ophthalmological clinics.

\section{CONCLUSION}

This study shows that the rate of ophthalmological consultation and the rate of DEN use can be increased by the awareness campaign by HCUNs. In the future, the development and utilization of a new DEN that includes more detailed patient information, and the spread of self-care guidance to patients by multidisciplinary health care professionals, will increase the consultation rate of MHD patients in Japan and reduce the incidence and progression of ocular diseases in MHD patients.

It is difficult to demonstrate the effectiveness of these results in countries with different healthcare systems from Japan. Longer- 
term studies involving other countries and other facilities are needed. However, the findings suggest that it is not detrimental for patients with diabetes in any country to have healthcare providers who are involved in their care to encourage and educate patients to seek ophthalmological care, and to use services such as the DEN. Based on the results of this study, we hope that more medical professionals around the world will become aware of patient involvement in healthcare and educate patients about ophthalmologic examinations for diabetic hemodialysis patients using tools such as the DEN, so that the quality of life of patients can be maintained and improved as much as possible.

\section{DATA AVAILABILITY STATEMENT}

The original contributions presented in the study are included in the article/supplementary material. Further inquiries can be directed to the corresponding author.

\section{REFERENCES}

1. Wong C, Wong T, Cheng C, Sabanayagam C. Kidney and Eye Diseases: Common Risk Factors, Etiological Mechanisms, and Pathways. Kindey Int (2014) 85(6):1290-302. doi: 10.1038/ki.2013.491

2. Lin H, Zheng C, Wu Y, Chang Y, Chen J, Liang C, et al. Diabetic Retinopathy as a Risk Factor for Chronic Kidney Disease Progression: A Multicenter Case-Control Study in Taiwan. Nutrients (2019) 11(3):509. doi: 10.3390/nu1130509

3. Park H, Lee Y, Cho A, Han C, Noh J, Shin Y, et al. Diabetic Retinopathy is a Prognostic Factor for Progression of Chronic Kidney Disease in the Patients With Type 2 Diabetes Mellitus. PloS One (2019) 14(7):e00220506. doi: 10.1371/journal.pone.0220506

4. Sabanayagam C, Chee M, Banu R, Cheng C, Lim S, Tai E, et al. Association of Diabetic Retinopathy and Diabetic Kidney Disease With All-Cause and Cardiovascular Mortality in a Multiethnic Asian Population. JAMA Netw Open (2019) 2(3):e191540. doi: 10.1001/jamanetworkopen.2019.1540

5. Dejesus E, Bejjanki H, Koratala A. Keep an Eye on the Eye Symptoms of Your Dialysis Patient. Am J Med (2019) 132(1):9-10. doi: 10.1016/ j.amjmed.2018.08.022

6. Mullaem G, Rosner M. Ocular Problems in the Patient With End-Stage Renal Disease. Semin Dial (2012) 25(4):403-7. doi: 10.1111/j.1525-139X.2012.01098.x

7. Pahor D. Retinal Light Sensitivity in Haemodialysis Patients. Nat Eye (2003) 17(2):177-82. doi: 10.1038/sj.eye.6700292

8. Ohguro N, Matsuda M, Fukuda M. Corneal Endothelial Changes in Patients With Chronic Renal Failure. Am J Ophthalmol (1999) 128, 2:234-36. doi: 10.1016/S0002-9394(99)00086-0

9. Muller M, Schonfeld C, Grammar T, Kohnne T, Drechsler C, Genser B, et al. Risk Factors for Retinopathy in Hemodialysis Patients With Type 2 Diabetes Mellitus. Nat Sci Rep (2020) 10(1):14158. doi: 10.1038/s41598-020-70998-9

10. Ciulla T, Amador A, Zinman B. Diabetic Retinopathy and Diabetic Masular Edema: Pathophysiology, Screening, and Novel Therapies. Diabetes Care (2003) 26(9):2653-64. doi: 10.2337/diacare.26.9.2653

11. Powers A. Diabetes Mellitus, Ophthalmologic Complications of Diabetes Mellitus. Harrison's Principles Internal Med (2008) 17:2287.

12. Hanafusa N, Fukagawa M. Global Dialysis Perspective: Japan. Kidney360 (2020) 1(5):416-9. doi: 10.34067/KID.0000162020

13. Tsukamoto Y. End-Stage Renal Disease (ESRD) and its Treatment in Japan. Nephrol Dialysis Transplant (2008) 23(8):2447-50. doi: 10.1093/ndt/gfn232

14. Funatsu H, Hori S. Present Status of Ophthalmological Care for Diabetic Patients in Japan. Jpn J Ophthalmol (2000) 44(1):75-81. doi: 10.1016/s00215155(99)00144-6

\section{ETHICS STATEMENT}

The studies involving human participants were reviewed and approved by Toyu Medical Research Ethics Review Committee. Written informed consent for participation was not required for this study in accordance with the national legislation and the institutional requirements. Informed consent was received from patients for the submissionof this manuscript to an academic journal.

\section{AUTHOR CONTRIBUTIONS}

MK, MT, AS, NI, and MF contributed to conception and design of the study. NS, MA, and EK organized the database. MK, MT, and NS performed the statistical analysis. MK and MT wrote the manuscript. All authors contributed to manuscript revision, read, and approved the submitted version.

15. Chen C, Herring J, Chen A. Managing Diabetic Retinopathy: The Partnership Between Ophthalmologist and Primary Care Physician. J Miss State Med Assoc (1995) 36(7):201-8.

16. Marrero DG, Moore PS, Langefeld CD, Clark CMJr. Patterns of Referral and Examination for Retinopathy in Pregnant Women With Diabetes by Primary Care Physicians. Ophthalmic Epidemiol (1995) 2(2):93-8. doi: 10.3109/ 09286589509057088

17. Goodkin D, Bragg-Gresham J, Koenig K, Wolfe R, Akiba T, Andreucci V, et al. Association of Comorbid Conditions and Mortality in Hemodialysis Patients in Europe, Japan, and the Practice Patterns Study (DOPPS). J Am Soc Nephrol (2003) 14(12):3270-77. doi: 10.1097/01.ASN.0000100127.54107.57

18. Saran R, Bragg-Gresham JL, Rayner HC, Goodkin DA, Keen ML, Van Dijk PC, et al. Nonadherence in Hemodialysis: Associations With Mortality, Hospitalization, and Practice Patterns in the DOPPS. Kidney Int (2003) 64 (1):254-62. doi: 10.1046/j.1523-1755.2003.00064.x

19. Carman KL, Dardess P, Maurer M, Sofaer A, Adams K, Bechtel C, et al. Patient and Family Engagement: A Framework for Understanding the Elements and Developing Interventions and Policies. Health Affairs (2013) 32(2):223-31. doi: 10.1377/hlthaff.2012.1133

20. Frampton SB, Guastello S, Hoy L, Naylor M, Sheridan S, Johnston-Fleece M. Harnessing Evidence and Experience to Change Culture: A Guiding Framework for Patient and Family Engaged Care. Washington, DC: National Academy of Medicine. Available at: https://nam.edu/harnessingevidence-and-experience-to-change-culture-a-guiding-framework-forpatient-and-family-engaged-care/ (Accessed January 31, 2017). doi: $10.31478 / 201701 \mathrm{f}$

21. Castro EM, Regenmortel TV, Vanhaecht K, Sermeus W, Van Hecke A. Patient Empowerment, Patient Participation and Patient-Centeredness in Hospital Care: A Concept Analysis Based on a Literature Review. Patient Educ. Couns (2016) 99:1923-39. doi: 10.1016/j.pec.2016.07.026

22. Maurer M, Dardess P, Carman KL, Frazier K, Smeeding L. Guide to Patient and Family Engagement, Contract Final Report: Environmental Scan ReportAHRQ Publication No.12-0042-EF. Rockville, MD: Agency for Healthcare Research and Quality (2012). Available at: https://www.ahrq. gov/research/findings/final-reports/ptfamilyscan/index.html.

23. Siminerio LM. Implementing Diabetes Self-Management Training Programs: Breaking Through the Barriers in Primary Care. Endocr Pract (2006) 12 (1):124-30. doi: 10.4158/EP.12.S1.124

24. Brink SJ, Miller M, Moltz KC. Education and Multidisciplinary Team Care Concepts for Pediatric and Adolescent Diabetes Mellitus. J Pediatr Endocrinol. Metab (2002) 15(8):1113-30. doi: 10.1515/jpem. 2002.15.8.1113 
25. The Japanese Society for Dialysis Therapy (2020). Available at: https://www. jsdt.or.jp/english/2426.html.

Conflict of Interest: The authors declare that the research was conducted in the absence of any commercial or financial relationships that could be construed as a potential conflict of interest.

Publisher's Note: All claims expressed in this article are solely those of the authors and do not necessarily represent those of their affiliated organizations, or those of the publisher, the editors and the reviewers. Any product that may be evaluated in this article, or claim that may be made by its manufacturer, is not guaranteed or endorsed by the publisher.

Copyright (ㄷ 2022 Kimura, Toyoda, Saito, Abe, Kato, Sugihara, Ishida and Fukagawa. This is an open-access article distributed under the terms of the Creative Commons Attribution License (CC BY). The use, distribution or reproduction in other forums is permitted, provided the original author(s) and the copyright owner(s) are credited and that the original publication in this journal is cited, in accordance with accepted academic practice. No use, distribution or reproduction is permitted which does not comply with these terms. 